\title{
APPLYING THE THEORY OF PLANNED BEHAVIOUR TO ACCOUNT FOR STUDENTS' CHOICE OF A TARGET ACCENT (PART 2)
}

Keywords: theory of planned behaviour, language attitudes, attitude-behaviour relations, target pronunciation model

\begin{abstract}
Bearing in mind the importance of attitude in sociolinguistic research and its huge theoretical potential for accounting for various language behaviours, it is surprising to see numerous misconceptions concerning this construct and its conceptualization as well as criticism as to its role in predicting and explaining speech behaviour (cf., for instance, Cargile, Giles 1997: 195; Edwards 1999: 109; Ladegaard 2000: 229-230; Garrett 2001: 630; Soukup 2012; Taylor, Marsden 2014). The author claims that attitude research can still prove very insightful and helpful in sociolinguistic theory building, but to do so, one needs to reconceptualize attitude along the reasoned action approach on the foundations of which the theory of planned behaviour rests. The theory posits that attitude is one of the three general predictors having a sufficient explanatory and predictive power in the case of most human behaviours. The major goal of the present article is to report on a study attempting to apply the theory of planned behaviour to explain why students of English being given an alternative to choose either an English or American accent as a target model to learn opt for one and not the other. The second goal of the article is to discuss the role of language attitudes in determining students' decisions. Part 2 of the article elaborates on the main study as well as includes a brief discussion followed by suggestions for further research.
\end{abstract}




\section{The main study - introduction}

The main study was conducted with a view to answering two research questions concerning respectively: (1) the assessment of the potential of the three TPB variables (predictors) for explaining a language-related behaviour and (2) the actual importance of attitude in determining students' behaviour. To specify, the study consisted in establishing the strength and nature of the correlations between the three variables and students' choices of the target accents: British (Received Pronunciation - RP) and American (General American - GA) ones. Since the theory of planned behaviour posits that individuals behave largely the way they do because of their attitudes, social pressure and perceived behavioural control, the three predictors were hypothesized to offer a valuable insight into the causes of students' course enrolment decisions and, in this way, account for their choices. It was taken that the greater the differences in the values assumed by a particular variable with respect to the two accents (for instance, attitude to GA vs. attitude to RP), the more likely it was that this variable impacted on students' choices. In addition, more extreme values of variables as opposed to those "more neutral" were assumed to point to their greater role in influencing students' decisions. Importantly, it was also presupposed that in some cases a decision to learn a given target accent could have resulted from very low values assumed by the variables related to the declined pronunciation model rather than a very favourable evaluation of the chosen accent.

When it comes to respondents, they were first-year students from the English Department at Adam Mickiewicz University. At the very beginning of the first semester, they could choose between the two accents that were taught there: American English and British English. The accents may be thought to correspond, respectively, to what has been labeled as Received Pronunciation and General American, i.e. models characterized by being made up of standard pronunciation features of the two varieties of English. Because the percentage of students who enrolled on the British pronunciation course was higher (around 60\%), it can be assumed that this accent enjoyed a greater popularity among the population of students. This state of affairs translated into a situation in which there were altogether more "British" groups than "American" ones. The majority of respondents could be regarded as young adults, highly motivated aspirers with over 10-year experience of English language learning who had achieved at least a B2 level of competence when entering the university. All of the respondents were asked about their perceptions of their speaking a certain accent and not simply about their decontextual perceptions of the accents in general. In this way, all questions were made relevant to their enrolment decision and subsequent language-related behaviour (learning and speaking this accent).

As regards the measurement instrument, it took the form of a written questionnaire distributed to students during their regular classes. It was prepared after conducting two pilot studies the aim of which was a selection of the most relevant semantic-differential scales. The final number of questionnaires accepted 
for a statistical analysis amounted to 53 forms from the "British" and 44 from the "American" groups. The theory of planned behaviour offers two methods of measuring attitude, subjective norm (SN) and perceived behavioural control (PBC). The one applied in this research consisted in constructing relevant scales probing into specific subcomponents of the three behavioural predictors (for details, see Ajzen 2002). ${ }^{1}$ Attitude here included adjectival pairs that were classified as experiential (attractive - unattractive, pleasant - unpleasant, interesting - uninteresting, satisfying - dissatisfying, natural - unnatural), instrumental (useful - useless, advantageous - disadvantageous, helpful - unhelpful) and general (e.g. the good bad scale). Subjective norm, in turn, encompassed injunctive and descriptive components. The injunctive one related to students' perception of what they should do because of other people's opinions (e.g. the one of parents, teachers and peers) or because of a conviction that something was expected of them. The descriptive item concerned their personal belief about the general popularity of a given accent, i.e. which one was more commonly used and learnt by people in Poland, especially by those who were important to the respondents or those who could be their role models. The components of PBC pertained to students' "sense of self-efficacy with respect to performing" a given behaviour and his/her perceived control over doing it (controllability) (Ajzen 2002: 7). In this study, the former concerned students' perception of their own aptitude for learning the accents and the latter related to their perception of the ease in doing so thanks to the accessibility of teaching materials, well-qualified staff, contacts with native speakers and opportunities for travel to the country where the accent was spoken.

All of the three variables were measured by means of seven-point, semanticdifferential scales ${ }^{2}$ in which the value 7 was given to the most favourably perceived items constituting a given variable, 1 to those indicating the least favourable perception and 4 to neither favourable nor unfavourable perception. In order to avoid automaticity in students' responses, a decision was made to vary the most favourable end-points of the scale so that they sometimes came on the left and sometimes on the right side of it. Attitude was measured by means of 9 bipolar scales whereas subjective norm and perceived behavioural control by two (one for each subcomponent). This was largely due to the greater complexity of the construct of attitude in comparison to the other two predictors. The statistical significance of the data presented in Tables 4 and 5 was verified by a T-test for dependent and independent variables. The level of statistical significance was established on the level of $\alpha=0.05$. Consequently, a given difference in students' perceptions of the two varieties of English can be regarded as statistically significant for $\alpha>p$.

1 Alternatively, the variables can be measured by means of relevant normative, control and attitudinal beliefs (Ajzen 1991).

2 The whole questionnaire was in Polish and the scales concerning attitude to speaking a given accent were the following: przyjemne - nieprzyjemne, satysfakcjonujące - niesatysfakcjonujące, ciekawe - nieciekawe, dobre - złe, przydatne - nieprzydatne, naturalne - nienaturalne, użyteczne - nieużyteczne, atrakcyjne - nieatrakcyjne, korzystne - niekorzystne. 


\section{Presentation of results}

To begin with, it must be reiterated that the following analysis of research results is based on an assumption that the more extreme evaluation of a given scale and, by extension, of a given predictor there was, the more likely it was to have impacted on students' choice of a pronunciation model to learn. Accordingly, when comparing the differences in the values of particular scales constituting attitude (see Table 4), one can come to a conclusion that experiential items (scales 1, 2, 3, 6, 8) of attitude contributed the most to students' from the "American" groups favourable evaluation of American English and their unfavourable perception of British English. This is especially true about the scales referring to pleasantness (the difference reached - 3.32) and naturalness (4.36). As regards the instrumental component (items 5, 7, 9), one can easily discern that students from "American" groups believed that their speaking British English would be for them only slightly unhelpful, useless and disadvantageous (values close to the "neutral" value of 4) and that American accent would be for them in this respect quite helpful, useful and advantageous. As for the influence of social pressure (SN) on the students' choice of a target accent, it seems that the injunctive component did not contribute to their perception of social pressure to speak either of the two accents. We can draw such a conclusion because there is no a statistically significant difference in perceiving which behaviour (speaking which accent) was expected of them. Despite this fact, the students did believe that there were slightly more people in their surroundings who spoke British English rather than American English (descriptive component). In this case, the difference proves to be statistically significant. Yet, it seems highly unlikely that subjective norm was one of the major determinants of these students' decision because the general mean values of $\mathrm{SN}$ indicate that the students did not perceive the two accents as much different in this respect. As regards perceived behavioural control, the data indicate that the students considered mastering American English, as opposed to learning British English, to be much easier for them (self-efficacy). When it comes to controllability, the differences were not so great but they were still quite noticeable and statistically significant. The general values also suggest that students must have taken PBC into account when opting for a given target accent.

As for attitude in the "British" groups, three scales (see Table 5) pointed to extremely different evaluations of the two accents. The scales were pleasant - unpleasant (the difference reached 3.51), satisfying - dissatisfying (3.75) and attractive - unattractive (3.93). Unexpectedly, students from the "British" groups did not consider speaking their chosen accent as something very natural for them and both accents, in fact, had surprisingly similar values (yet, the difference was still statistically significant). What really mattered for this group of students was the perceived attractiveness of the accent as well as the satisfaction and pleasure derived from speaking it. Regarding the instrumental component of attitude (scales 5, 7, 9), it seems that since the differences in evaluations of the two accents were not great (notwithstanding, statistically significant), the items may have contributed to the students' decision to learn RP only moderately. All in all, the global mean values of attitude indicate 


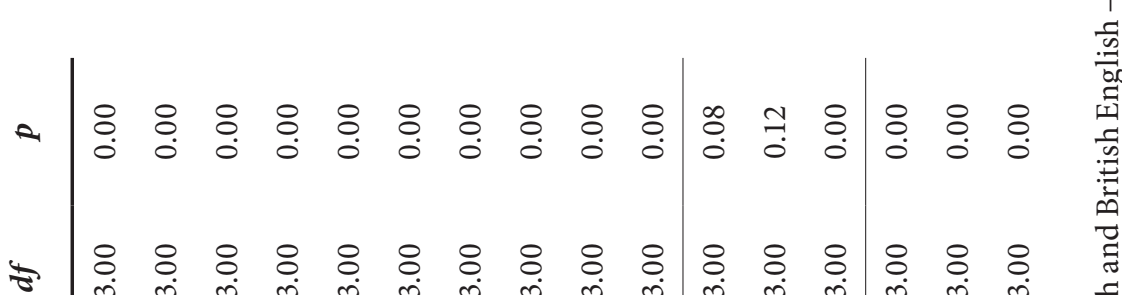

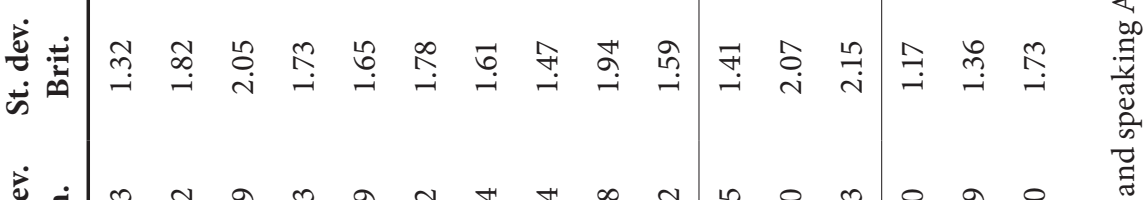

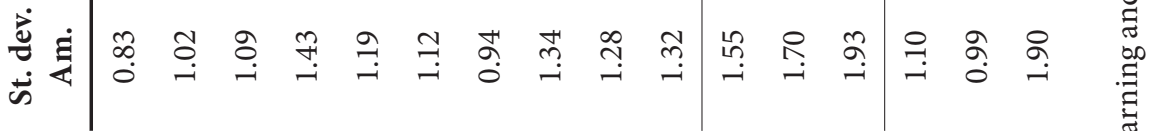

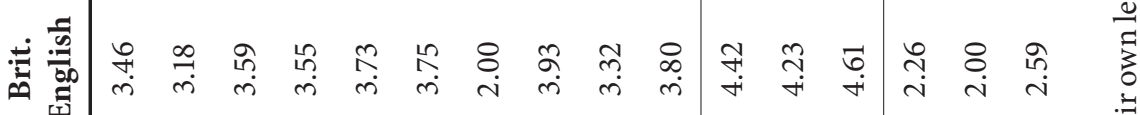

글

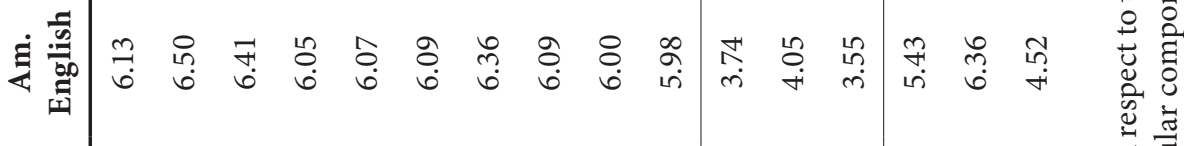

章

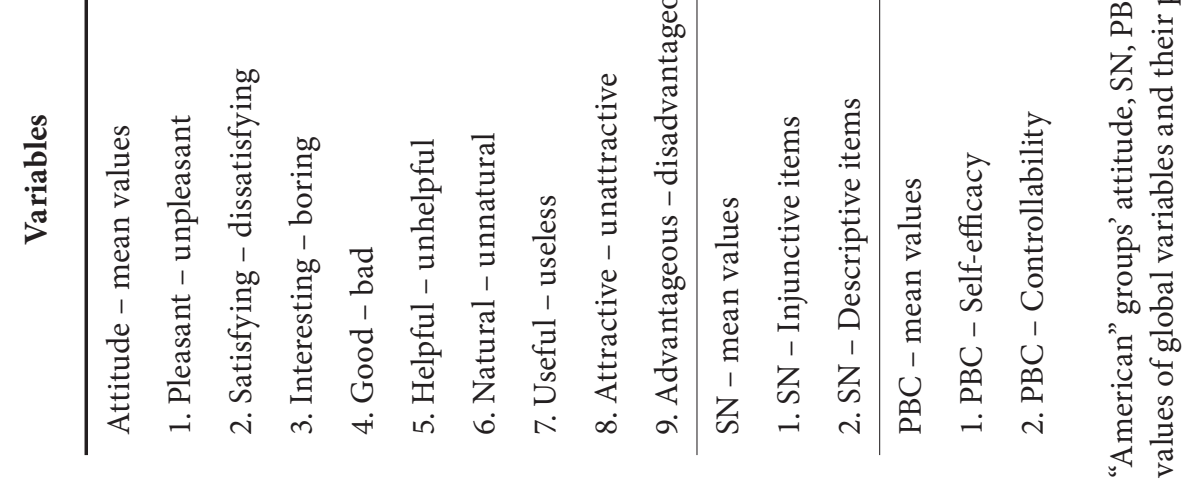




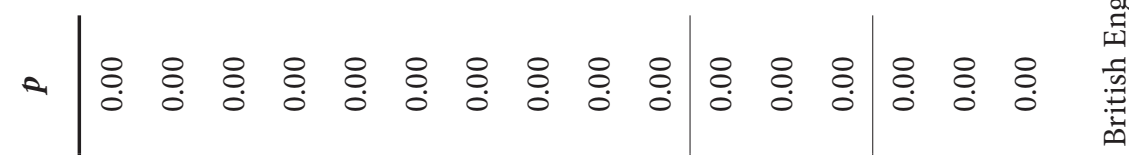


that students from the "British" groups perceived speaking British English very favourably and American English slightly unfavourably.

An analysis of the importance of subjective norm suggests that the "British" groups did feel some social pressure to opt for the British accent as their target pronunciation model (especially, when it comes to the injunctive component). Regarding the perceived popularity of the two accents (the descriptive component), the discrepancies in the perception of British English and American English by this group of students proved very small; yet, they were still statistically significant. Interestingly, the mean values of the particular components of PBC show quite different patterns from the ones found in the "American" groups. Although these students considered their target accent to be easier to learn because of both their aptitude and some external conditions facilitating acquisition, they recognized their controllability over acquiring the pronunciation model to be higher and more important than self-efficacy. Another difference was that the values assumed by all PBC components were more moderate than in the case of their perception by the "American" groups.

\section{Discussion of results}

The first research question posed in the study was the one asking why students of English being given an alternative to choose either the British or American accent as a target one to learn opt for one and not the other. The theory of planned behaviour applied in this study took into account three variables: attitude, subjective norm (SN) and perceived behavioural control (PBC) (see Table 6 and Figure 2). Even a cursory glance at Figure 2 can help one notice some highly interesting tendencies. First of all, both groups of students perceived the accent they decided to learn almost just as favourably and the one they declined just as unfavourably. Moreover, the degree of discrepancies in the attitudinal evaluation of the two accents by each group was also highly corresponding, which suggests that attitude exerted a very similar influence on their choices of target pronunciation models. Interestingly, one can discern another surprising similarity, namely, the one concerning students' perceived social pressure with respect to the two accents (see Figure 2). It is intriguing that both groups evaluated the general social pressure towards each of the accent to be almost on the same level (the differences in their perceptions are not statistically significant - see Table 6). To specify, all students felt that there was some moderate pressure on learning and using the British accent and that it was slightly unlikely that there was any pressure of this kind in the case of the American accent. This unanimity in their general perception seems to indicate that it possible for different groups to have dissimilar attitudes and yet, at the same time, to perceive the social pressure towards a given behaviour similarly. It also implies that positive attitudes in the case of these groups did not rather stem from their perception of subjective norm. Importantly, it must be emphasized that even if there were some similarities in the perception of particular components of subjective norm, 
one can still notice some slight discrepancies in the two groups. To specify, both groups perceived the injunctive and descriptive aspects to be higher in the case of the British accent (see Tables 4 and 5). Nevertheless, whereas in the British groups the injunctive component with respect to the British accent achieved the highest value, in the American groups it was the descriptive component with respect to the British accent that proved the most significant.

\begin{tabular}{lccccccc}
\multicolumn{1}{c}{ Variables } & $\begin{array}{c}\text { 'Am.' } \\
\text { group }\end{array}$ & $\begin{array}{c}\text { 'Br.' } \\
\text { group }\end{array}$ & $\begin{array}{c}\text { St. dev. } \\
\text { Am. }\end{array}$ & $\begin{array}{c}\text { St. dev. } \\
\text { Brit. }\end{array}$ & $\boldsymbol{T}$ & $\boldsymbol{d f}$ & $\boldsymbol{p}$ \\
\hline $\begin{array}{l}\text { 1. Attitude towards } \\
\text { Am. English }\end{array}$ & 6.13 & 3.56 & 0.83 & 1.21 & 11.91 & 95 & 0.00 \\
$\begin{array}{l}\text { 2. Attitude towards } \\
\text { Brit. English }\end{array}$ & 3.46 & 6.24 & 1.32 & 0.61 & -13.63 & 95 & 0.00 \\
$\begin{array}{l}\text { 3. SN with respect } \\
\text { to Am. English }\end{array}$ & 3.74 & 3.53 & 1.55 & 1.08 & 0.78 & 95 & 0.43 \\
$\begin{array}{l}\text { 4. SN with respect } \\
\text { to Brit. English }\end{array}$ & 4.42 & 4.52 & 1.41 & 1.34 & -0.35 & 95 & 0.72 \\
$\begin{array}{l}\text { 5. PBC with respect } \\
\text { to Am. English }\end{array}$ & 5.43 & 3.50 & 1.10 & 1.38 & 7.52 & 95 & 0.00 \\
$\begin{array}{l}\text { 6. PBC with respect } \\
\text { to Brit. English }\end{array}$ & 2.26 & 4.90 & 1.17 & 1.36 & -10.15 & 95 & 0.00
\end{tabular}

Table 6. "American" and "British" groups' perceptions of attitudes, SN and PBC concerning the two accents - global variables

As regards the perceived behavioural control, both groups believed that they were much more likely to better acquire the accent they chose (see Table 6). However, it is clear that this variable must have played a much greater role on the choice of students from the "American" groups since their evaluations of the two pronunciation models were far more extreme. This was especially so in the case of their self-efficacy (compare Tables 4 and 5). Surprisingly, even students from the "British" groups did not consider the ease of learning the British accent as compared with the American one to be decidedly greater. Possibly, this may be caused by a general perception of the British culture and, by extension, British accent as more sophisticated (and, thus, more complex). American culture, in contrast, seems to be regarded as lower and cruder which may translate into a perception of American accent as an easier pronunciation model to learn. All in all, there is rather no one easy answer to the first research question. The "American" groups decided to learn the American accent because of very positive attitude and perceived behavioural control towards this pronunciation model. Their decision was not changed even despite their feeling a slight social pressure to choose the British accent. To conclude, students from the "British" groups opted for the British model because of all the three reasons 
(attitude, SN and PBC). However, only attitude assumed very high values and the other two variables only very moderate ones (see Table 6). By way of conclusion, one can also say that the variables of the theory of planned behaviour seem to have provided considerable insight into the major reasons for students' choices and constituted a measurement instrument sensitive enough to point to the differences between the two groups.

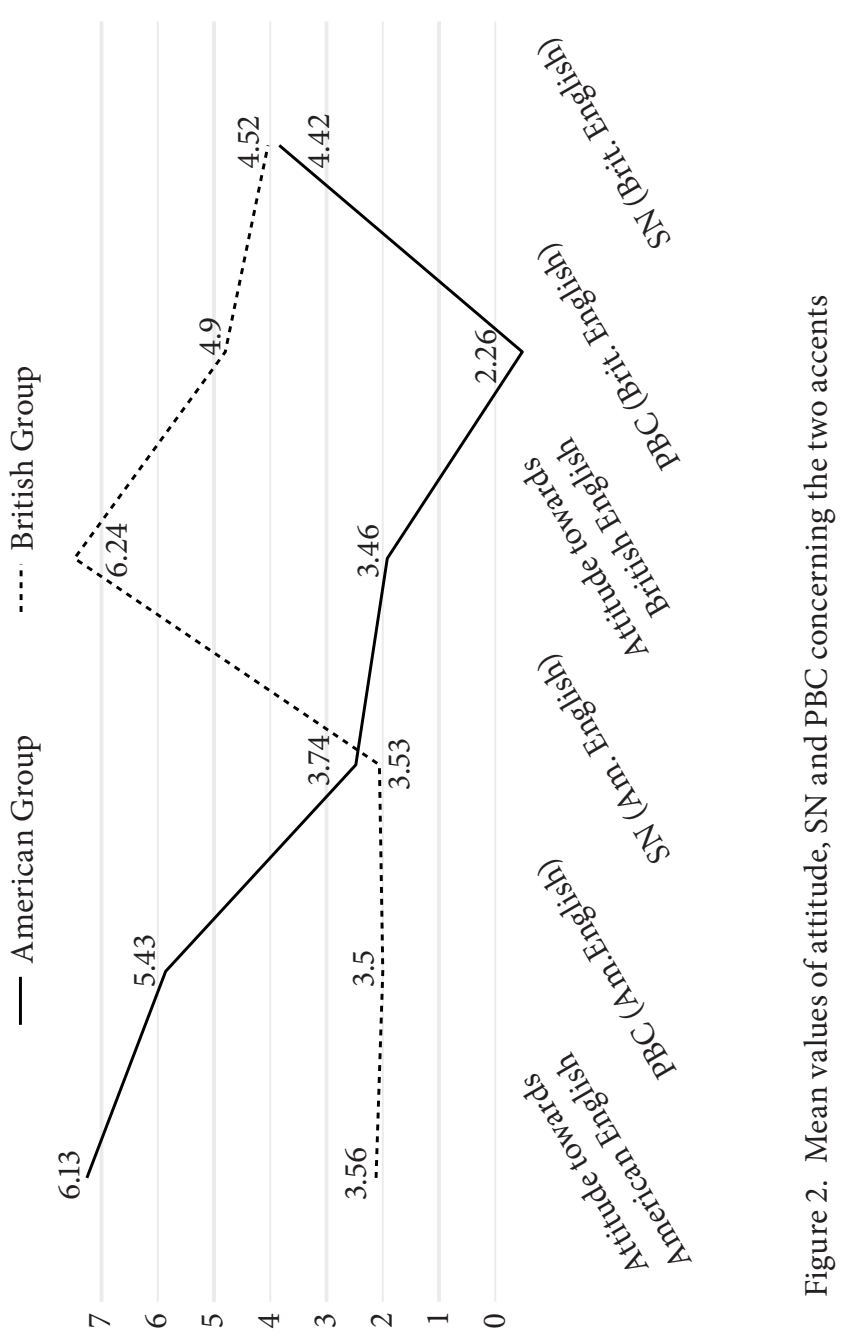

The second research question concerned the actual role of language attitudes in determining students' choice of a target accent. The most important finding resultant from the above analysis is that attitude appears to have been a factor which determined students' decisions to the largest extent. This is obviously so because of the fact that students' evaluations of the two accents revealed the greatest discrepancies when it comes to their attitudinal reactions (see Table 6). With the exception of students' 
from "American" groups perception of PBC (and especially self-efficacy), all other variables assumed only slightly favourable or unfavourable values. This seems to suggest that attitude played a major role in all students' decisions and that only for students from "American" groups their aptitude was a factor they may have seriously taken into account. Interestingly, the mean values of the three variables seem to indicate that people may agree on the perceived social pressure to perform a given behaviour (or not to perform it); yet, they may have different attitudes towards a given action and, consequently, manifest different behavioural patterns consistent with their attitudes (see Table 6). Furthermore, results from the "British" groups seem to indicate that it is possible to develop a favourable attitudinal evaluation of a given behaviour even despite having rather "neutral" perception of one's perceived behavioural control (both self-efficacy and controllability). All of this seems to point to some kind of independence (at least potential independence) of the three variables: attitude, SN and PBC. The last valuable finding is that students' favourable attitudes to the accents they opted for were determined by different adjectival scales in the case of both groups. For "American" groups, these were pleasant - unpleasant (6.50), satisfying - dissatisfying (6.41), natural - unnatural (6.36) and for "British" groups satisfying - dissatisfying (6.79), attractive - unattractive (6.74) and pleasant - unpleasant (6.51). It is very surprising that for "British" groups their learning and speaking "British" accent was perceived as something only moderately natural (4.96). This could be caused by a far less widespread presence of the British culture in the popular media in Poland which is dominated by American productions and the American variety of English (Przygoński 2012, 2016). As a result of that, the British accent is likely to be regarded as more distant, more sophisticated due to the associations it evokes with the rich British history and the royal family. In conclusion, in the case of the behaviour under investigation it was attitude that proved a factor that impacted on students' choice of a pronunciation model to the largest extent.

\section{Conclusions and limitations}

The major aim of this research was accounting for speech behaviour by means of applying the theory of planned behaviour, a theory suggesting that human action can be predicted and explained by three different factors whose individual saliency and importance in determining a specific behaviour can vary across contexts and populations. The results of the study clearly indicate that attitude, due to its extreme values, seemed to have a decisive say in the students' choice of the target accent. It emerged as one of the most important factors which had an influence on all students' decisions to learn to speak a given pronunciation model. Both groups perceived the accent they chose very favourably and the other "neutrally" or slightly unfavourably. Accordingly, attitude, as conceptualized in this research project, allows for greater contextual sensitivity and considerably increases the predictive and explanatory power of the concept. The study also pointed to a moderate positive importance of other factors on their decisions, i.e. social pressure and perceived 
behavioural control. Importantly, it was shown that language attitudes conceptualized in line with the reasoned action approach can offer valuable insight in the case of at least some speech behaviours and some research groups.

Despite considerable effort put into the methodological correctness of the present study and its theoretical grounding, the research project is not free of limitations which should be seriously taken into account when designing future investigations. The following list should be treated as a lodestar pointing to the areas requiring further improvement and development: (1) an application of more advanced statistical tools probing into the combined effects of predictors or their specific subcomponents upon language-related behaviours, (2) developing a more ethnographic-oriented approach to selecting measurement scales for other predictor variables, i.e. for subjective norm and perceived behavioural control, (3) designing a research project the aim of which will be examining the prognostic potential of the theory of planned behaviour in language-related behaviours rather than its explanatory power.

\section{References}

Ajzen I. 1991. The theory of planned behavior. - Organizational Behavior and Human Decision Processes 50: 179-211.

Ajzen I. 2002. Constructing a theory of planned behavior questionnaire: Conceptual and methodological considerations. [http://www.people.umass.edu-/aizen/pdf/tpb.measurement.pdf].

Cargile A.C., Giles H. 1997. Understanding language attitudes: Exploring listener affect and identity. - Language and Communication 17.3: 195-217.

Edwards J. 1999. Refining our understanding of language attitudes. - Journal of Language and Social Psychology 18.1: 101-110.

Garrett P. 2001. Language attitudes and sociolinguistics. - Journal of Sociolinguistics 5.4: $626-631$.

Ladegaard H.J. 2000. Language attitudes and sociolinguistic behavior: Exploring attitudebehavior relations in language. Journal of Sociolinguistics 4.2: 214-233.

Przygoński K. 2012. Sociolinguistic aspects of the functioning of English in post-1989 Poland. Frankfurt am Main.

Przygoński K. 2016. Political transformation as a trigger for a sociolinguistic resolution in post-socialist Poland: English and its rising power. - Sloboda M., Laihonen P., Zabrodskaja A. (eds.). Sociolinguistic transition in former eastern bloc countries. Two decades after the regime change. Frankfurt am Main: 115-139.

Soukup B. 2012. Current issues in the social psychological study of 'language attitudes': Constructionism, context, and the attitude-behavior link. - Language and Linguistics Compass 6.4: 212-224.

Taylor F., Marsden E.J. 2014. Perceptions, attitudes, and choosing to study foreign languages in England: An experimental intervention. - The Modern Language Journal 98.4: 902-920. 
